\title{
A Case of Incidentally Found Bilateral Retroperitoneal Cavernous Hemangiomas
}

\author{
Shunsuke Yui ${ }^{1}$, Masayoshi Zaitsu' ${ }^{1}$ Koji Mikami ${ }^{1}$, Yuta Takeshima ${ }^{1}$, Akiko Tonooka ${ }^{2}$, \\ Toshimasa Uekusa ${ }^{2}$, Takumi Takeuchi ${ }^{1}$ \\ ${ }^{1}$ Department of Urology, Kanto Rosai Hospital, Kawasaki, Japan \\ ${ }^{2}$ Pathology, Kanto Rosai Hospital, Kawasaki, Japan \\ E-mail: takeuchit@abelia.ocn.ne.jp \\ Received July 5, 2011; revised July 31, 2011; accepted August 9, 2011
}

\begin{abstract}
A 59-year-old male was admitted to our hospital because of incidentally found right retroperitoneal tumor. He had undergone removal of a hemangioma in the left oral cavity four years before. An abdominal CT scan performed in our hospital revealed poorly enhanced bilateral retroperitoneal tumors adjacent to kidneys. Those tumors were of low signal intensity on T1-weighted images and high on T2-weighted images by magnetic resonance imaging. The right retroperitoneal tumor of $2.5 \mathrm{~cm}$ in size was surgically removed and histopathological examination indicated cavernous hemangioma. The smaller left retroperitoneal tumor of $1.1 \mathrm{~cm}$ in size was left untouched to be followed up, as we supposed that it has the same benign pathology. There have been no previous cases of retroperitoneal cavernous hemangioma as a presentation of multiple hemangiomas.
\end{abstract}

Keywords: Hemangioma, Bilateral, Retroperitoneum

\section{Introduction}

Primary retroperitoneal tumors just account for $0.2 \%$ of all tumors in a old article published in 1954 [1] and there are no available reports definitely describing the recent prevalence of retroperitoneal tumors. Braasch et al. collected 101 cases of retroperitoneal tumor from the literature and reported in 1967 that more than $80 \%$ of cases were malignant and symptomatic [2]. Nowadays benign, smaller, and less symptomatic retroperitoneal tumors must be diagnosed with the improvement and prevalence of advanced imaging modalities as CT scan, magnetic resonance imaging, and ultrasonography. Nakajima et al. reported in 1997 that $10(40 \%)$ out of 25 retroperitoneal tumors were malignant [3], thus the percentage of malignancy among all diagnosed retroperitoneal tumors seems decreased than before.

Retroperitoneal hemangioma is very uncommon, while the true incidence of that disorder among retroperitoneal tumors is basically unknown, as most of them may be asymptomatic and remain undiagnosed. That there have been only few reports of retroperitoneal hemangioma diagnosed only with radiological imagings [4] may indicate that surgical procedure has been chosen for

both diagnostic and therapeutic purposes. Here we show a case of bilateral retroperitoneal cavernous hemangiomas which was difficult to be diagnosed before surgical extirpation.

\section{Case Report}

A 59-year-old male was admitted to our hospital because of incidentally found right retroperitoneal tumor. Tumor markers as well as regular laboratory data were unremarkable. Urine cytology was negative. An abdominal CT scan performed in our hospital revealed a right retroperitoneal tumor of $2.5 \mathrm{~cm}$ in size and a left retroperitoneal tumor of $1.1 \mathrm{~cm}$ in size adjacent to either kidney. Those tumors with speckled calcification were not enhanced so much both in early phase and late phase (Figure 1). By an abdominal magnetic resonance imaging, those tumors were of low signal intensity on T1weighted images and high on T2-weighted images (Figure 2). We have decided to extirpate the right retroperitoneal tumor using transperitoneal approach, because those tumors could not be determined to be of benign pathology by imaging studies. We did not adopt lararoscopic and retroperitoneoscopic surgery because of un- 
known pathology.

At operation, there was little adhesion between the tumor and the surrounding structures. The tumor was supposed to be originated from the vascular sheath of the right renal artery and there were small feeders from the sheath. The tumor was irregular on surface and reddish like a raspberry in color (Figure 3). We utilized renal arterial clump for a short time in order to reduce gradual bleeding from the tumor surface which occurred when the preparation of the tumor was initiated. The resected tumor was $3.0 \times 1.5 \times 1.5 \mathrm{~cm}$ in size. The cut section showed sponge-like appearance containing solid component (Figure 4).

Histology of the tumor (Figure 5) was diagnosed as cavernous hemangioma composed of elaborately interanastomosing vascular spaces of large dilated blood-filled vessels. Vascular channels with fibrous walls were lined by flattened endothelial cells which have no atypism. The smaller left retroperitoneal tumor of $1.1 \mathrm{~cm}$ in size was left untouched to be followed up, as we supposed that it has the same benign pathology.

\section{Discussion}

We have experienced a relatively rare case of bilateral retroperitoneal cavernous hemangiomas occurred in an elder man. Hemangioma is often found in infants, especially in the skin [5]. Hemangioma is histologically defined as a benign tumor or malformation made up of mature well-formed vessels usually lined by a single layer of endothelium and is classified into six categories, i.e. capillary hemangioma, cavernous hemangioma, venous hemangioma, epithelioid hemangioma, pyogenic
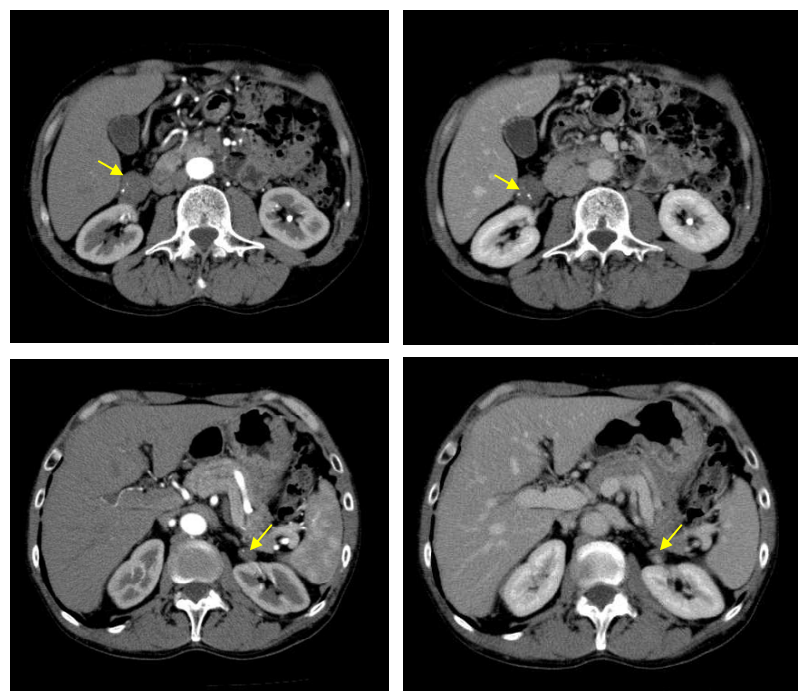

Figure 1. Example of a figure caption A CT scan demonstrating bilateral retroperitoneal tumors. Arrows indicate tumors.
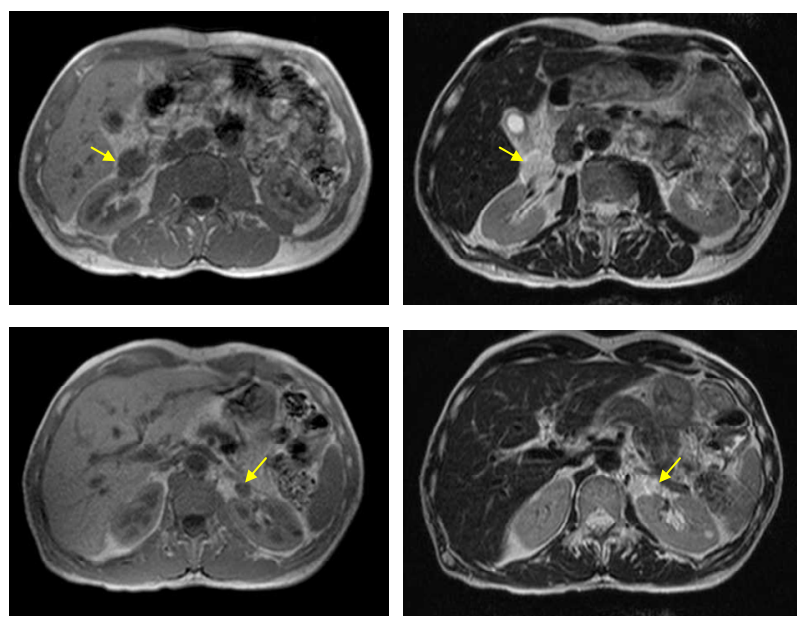

Figure 2. An MR imaging demonstrating bilateral retroperitoneal tumors. Arrows indicate tumors.

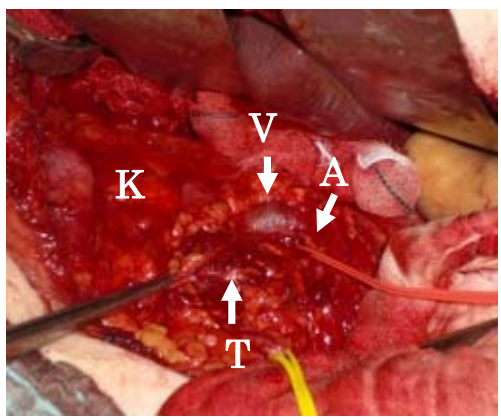

Figure 3. A photograph during operation. T: right retroperitoneal tumor, $A$ : taped right renal artery, $\mathrm{V}$ : right renal vein, $K$ : right kidney.

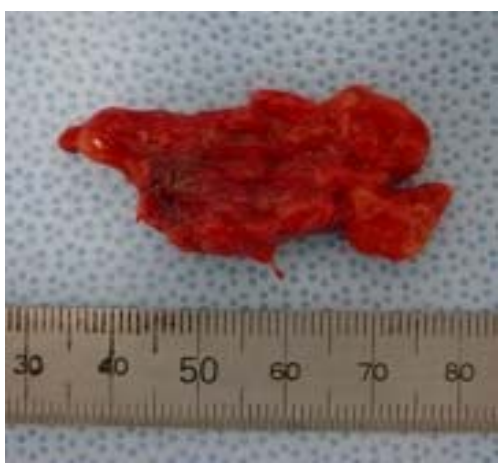

Figure 4. A macroscopic cut section of the right retropritoneal tumor.

granuloma, and acquired tirfted hemangioma [6]. Hemangioma is also known to be found in the oral region [7] as was observed in the present case.

In the present case, hemangiomas were multiple in bilateral retroperitoneal spaces as well as in the mouth. Multiple hemangiomas are often a part of established syndromes such as Sturge-Weber syndrome, Maffucci syndrome, Kasabach-Merritt syndrome, and von Hippel- 


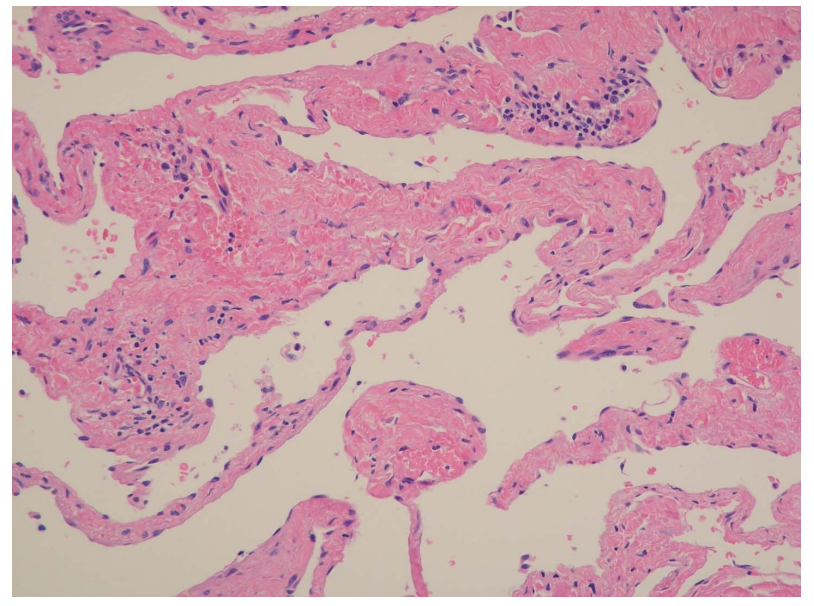

Figure 5. Hematoxylin and eosin staining of the right retroperitoneal tumor showing cavernous hemangioma.

Lindau disease, but this case did not seem to apply to them as it lacks in the characteristics of those disorders. There have been no previous cases of retroperitoneal cavernous hemangioma as a presentation of multiple hemangiomas.

Surgical removal of retroperitoneal tumors is indicated as long as the possibility of potential malignancy remains. Maximum diameter of malignant retroperitoneal tumors is reported to be larger than that of benign ones (11.45 \pm $1.90 \mathrm{~cm}$ vs $5.31 \pm 0.43 \mathrm{~cm}$ ) [3]. The tumor size of the present case was relatively small and the probability of malignancy was not very high in the view points of tumor size, then we might have followed up the interval change in the tumor size before indicating surgery. Additionally, retroperitoneal hemangiomas have a risk of rupture and bleeding [8] even they are histologically benign, leading to a rational of choosing surgical extirpation especially when they are large. Although we adopted open surgical approach, laparoscopic resection of retroperitoneal cavernous hemangioma has been reported $[9,10]$. It may be appropriate to remove retroperitoneal hemangiomas laparoscopically or retroperitoneoscopically if they are probably benign.

Besides surgical treatments, there are some options as corticosteroid therapy and radiotherapy in the treatment of hemangioma. While corticosteroid therapy is mainly a choice of treatments of capillary hemangioma [11], there is a report of unresectable $8 \times 5 \mathrm{~cm}$ retroperitoneal cavernous hemangioma diagnosed in a female neonate 20 days after birth and showing anemia and thrombocytopenia, which disappeared following oral corticosteroid therapy [12]. Radiotherapy is also given to treat symptomatic hemangiomas [13].

In the present case, the smaller left retroperitoneal tumor of $1.1 \mathrm{~cm}$ in size was left untouched to be followed up, as we supposed that it has the same benign pathology.
When the left retroperitoneal tumor grows larger enough, we may need to re-consider if he needs some treatment including surgery.

\section{References}

[1] G. T. Pack and E. J. Tabah, "Collective Review: Primary Retroperitoneal Tumors; a Study of 120 Cases," Surgery, Gynecology \& Obstetrics, Vol. 99, No. 3, 1954, pp. 209231.

[2] J. W. Braasch and A. B. Mon, "Primary Retroperitoneal Tumors," Surgical Clinics of North America, Vol. 47, No. 3, 1967, pp. 663-678.

[3] J. Nakashima, M. Ueno, K. Nakamura, M. Tachibana, S. Baba, N. Deguchi, H. Tazaki and M. Murai, "Differential Diagnosis of Primary Benign and Malignant Retroperitoneal Tumors,” International Journal of Urology, Vol. 4, No. 5, 1997, pp. 441-446. doi:10.1111/j.1442-2042.1997.tb00282.x

[4] N. Takaha, M. Hosomi, K. Sekii, S. Nakamori, K. Itoh, S. Sagawa, T. Kido, M. Satani, S. Kawamoto and K. Sato, "Retroperitoneal Cavernous Hemangioma: A Case Report,” Hinyokika Kiyo, Vol. 37, No. 7, 1991, pp. 725-728.

[5] B. A. Drolet, N. B. Esterly and I. J. Frieden, "Hemangiomas in Children," New England Journal of Medicine, Vol. 341, No. 3, 1999, pp. 173-181. doi:10.1056/NEJM199907153410307

[6] S. W. Weiss, "Histological Typing of Soft Tissue Tumours,” Vol. 2, Springer-Verlag, New York, 1994.

[7] N. Kawachi, “A Comparative Histopathological and Immunohistochemically Study of Capillary Hemangioma, Pyogenic Granuloma and Cavernous Hemangioma in the Oral Region: With Special Reference to Vascular Proliferation Factors,” International Journal of Oral-Medical Sciences, Vol. 9, No. 3, 2011, pp. 241-251. doi:10.5466/ijoms.9.241

[8] T. L. Forbes, "Retroperitoneal Hemorrhage Secondary to a Ruptured Cavernous Hemangioma,” Canadian Journal of Surgery, Vol. 48, No. 1, 2005, pp. 78-79.

[9] Y. S. Choi and H. K. Oh, "Laparoscopic Resection of a Retroperitoneal Hemangioma Arising from Ovarian Vessels,” Journal of Minimally Invasive Gynecology, Vol. 16, No. 6, 2009, pp. 778-780. doi:10.1016/j.jmig.2009.07.017

[10] R. N. P. Martín, J. E. Zarranz, C. V. F. Mdel, C. C. Redondo, J. A. Sesmero and J. Martinez-Sagarra, "Laparoscopic Resection of Retroperitoneal Venous Hemangioma,” Journal of Urology, Vol. 171, No. 1, 2004, p. 336.

[11] M. T. Edgerton, "The Treatment of Hemangiomas: With Special Reference to the Role of Steroid Therapy," Annals of Surgery, Vol. 183, No. 5, 1976, pp. 517-532. doi:10.1097/00000658-197605000-00009

[12] Y. Kasubuchi, T. Sawada and T. Nakamura, "Successful Treatment of Neonatal Retroperitoneal Hemangioma with Corticosteroid,” Journal of Pediatric Surgery, Vol. 8, No. 1, 1973, pp. 59-62. 


\section{doi:10.1016/0022-3468(73)90296-0}

[13] R. K. Aich, A. R. Deb, A. Banerjee, R. Karim and P. Gupta, "Symptomatic Vertebral Hemangioma: Treatment with Radiotherapy,” Journal of Cancer Research and Therapeutics, Vol. 6, No. 2, 2010, pp. 199-203.

doi:10.4103/0973-1482.65248 